\title{
Exact relativistic models of conformastatic charged dust thick disks
}

\author{
Gonzalo García-Reyes* \\ Departamento de Física, Universidad Tecnológica de Pereira, A. A. 97, Pereira, Colombia
}

We construct relativistic models of charged dust thick disks for a particular conformastatic spacetime through a Miyamoto-Nagai transformation used in Newtonian gravity to model disk like galaxies. Two simple families of thick disk models and a family of thick annular disks based on the field of an extreme Reissner-Nordström black hole and a Morgan-Morgan-like metric are considered. The electrogeodesic motion of test particles around the structures are analyzed. Also the stability of the particles against radial perturbation is studied using an extension of the Rayleigh criteria of stability of a fluid in rest in a gravitational field. The models built satisfy all the energy conditions.

* e-mail: ggarcia@utp.edu.co 


\section{INTRODUCTION}

Axially symmetric exact solutions of Einstein field's equations describing the field of a disk are important in astrophysics as models of certain stars, flat galaxies, accretion disks, the superposition of a black hole and a galaxy or an accretion disk as in the case of quasars, and in general relativity as sources of exact solutions of Einstein equations. Exact solutions representing static thin disks were first studied by Bonnor and Sackfield [1] and Morgan and Morgan [2,3]. Several classes of exact solutions of the Einstein equations corresponding to static thin disks have been obtained by different authors [4-10], while rotating thin disks were studied in [11, 12]. The exact superposition of a disk and a static black hole was first considered by Lemos and Letelier [13, 14]. Static thick disk models were considered by González and Leterier [15] and rotating thick disks by Voght and Letelier [16].

On the other hand, thin disks in presence of electromagnetic field have been discussed as sources for Kerr-Newman fields [17], conformastationary metrics [18], and magnetostatic axisymmetric fields [19 23]. Now, have been showed that gravitationally bound system such as stars, galaxies, and clusters of galaxies can be positively charged 24, 25], so that electric fields can also be important in the study of self-gravitating system not only from the merely theoretical point of view but also astrophysical. Thus, in presence of a pure electric field, conformastatic thin disks made of a charged perfect fluid have been considered in [26], and composed of electrically counterpoised dust [27 29], i.e. with the electric charge density equal to the mass density, in reference [30] for nonaxisymmetric planar matter distribution, in [31] for Morgan-Morgan-like fields and in [32] for the superposition of an extreme Reissner-Nordström black hole and a annular disk.

In this work we construct exact relativistic models of thick disks made of a charged dust fluid for a particular conformastatic spacetime through a Miyamoto-Nagai transformation used in Newtonian gravity to model disk like galaxies [33, 34], which adds to the previous electrostatic disks models an additional degree of reality, its thickness. Two simple families of thick disk models and a family of thick annular disks based on the field of an extreme ReissnerNordström black hole and a Morgan-Morgan-like solution are considered. The ring like matter distributions are generated applying a Kelvin transformation [37, 38]. The structures have no boundary but as the energy density decreases rapidly one can define a cutoff radius and, in principle, to consider such structures as finite.

The paper is organized as follows. In Sec. II we present the formalism to construct models of thick disks made of charged dust for a particular conformastic metric. We also analysis the electrogeodesic motion of charged test particles around the disks and the stability of the orbits against radial perturbation using an extension of the Rayleigh criteria of stability of a fluid in rest in a gravitational field. In Secs. III-V two simple family of charged dust thick disks and a family of thick annular disks are considered based on the field of an extreme Reissner-Nordström black hole and a Morgan-Morgan-like solution. Finally, in Sec. VI we summarize and discuss the results obtained.

\section{EINSTEIN-MAXWELL EQUATIONS AND DISKS}

We consider a conformastatic spacetime in cylindrical coordinates $(\varphi, R, z)$ and in the particular form

$$
d s^{2}=-(1-\psi)^{-2} d t^{2}+(1-\psi)^{2}\left(R^{2} d \varphi^{2}+d R^{2}+d z^{2}\right)
$$

For axially symmetric fields $\psi$ is function of the coordinates $R$ and $z$ only. The Einstein-Maxwell equations, in geometrized units such that $G=c=1$, are given by

$$
\begin{aligned}
R_{a b} & =8 \pi\left(T_{a b}^{\mathrm{m} a t}+T_{a b}^{\mathrm{e} m}\right) \\
\nabla_{b} F^{a b} & =4 \pi J^{a}
\end{aligned}
$$

where

$$
\begin{aligned}
T_{a b} & =\frac{1}{4 \pi}\left[F_{a c} F_{b}{ }^{c}-\frac{1}{4} g_{a b} F_{c d} F^{c d}\right], \\
F_{a b} & =A_{b, a}-A_{a, b} .
\end{aligned}
$$

The other symbols have the usual meaning, i.e., ( ), $a=\partial / \partial x^{a}, \nabla_{b}$ covariant derivate, etc.

For a charged dust distribution

$$
\begin{aligned}
T_{a b}^{\mathrm{m} a t} & =\rho V_{a} V_{b}, \\
A_{a} & =\delta_{a}^{0} \phi,
\end{aligned}
$$


where $\rho$ is the energy density, $V^{a}$ the four-velocity of the fluid, and $\phi$ electrostatic potential, the Einstein-Maxwell equations have solution in the case of spacetime (1) [27-29]

$$
\begin{aligned}
& \phi=k(1-\psi)^{-1}, \\
& \rho=\frac{\nabla^{2} \psi}{4 \pi(1-\psi)^{3}}, \\
& \sigma=k \rho,
\end{aligned}
$$

where $\sigma$ is the electric charge density and $k= \pm 1 . V^{a}$ is the timelike vector of the orthonormal tetrad (comoving observer) $\mathrm{e}_{(a)}^{b}=\left\{V^{b}, W^{b}, X^{b}, Y^{b}\right\}$, where

$$
\begin{array}{ll}
V^{a}=(1-\psi) \delta_{t}^{a}, & W^{a}=(1-\psi)^{-1} \delta_{\varphi}^{a} / R, \\
X^{a}=(1-\psi)^{-1} \delta_{R}^{a}, & Y^{a}=(1-\psi)^{-1} \delta_{z}^{a} .
\end{array}
$$

In the Newtonian limit $\psi \ll 1$, the expression relativistic (5b) reduces to the Poisson's equation and in absence of matter to the Laplace's equation.

Exact solutions of Einstein-Maxwell's equations representing the field of a disk of infinitesimal thickness immersed or not in a matter distribution can be obtained applying a Kuzmin-Toomre transformation [35, 36] $z \rightarrow a+|z|$, where $a$ is a positive constant, and models of thick disks using a Miyamoto-Nagai transformation which consists in changing in the Kuzmin-Toomre thin disks $|z| \rightarrow \zeta=\sqrt{z^{2}+b^{2}}$, where $b$ is a positive parameter. When this procedure is applied to a electrovacuum Einstein-Maxwell solution (5a) - (5c), we obtain a relativistic charged dust thick disk with energy density given by

$$
\rho=\frac{b^{2}\left(\zeta^{-1} \psi_{, \zeta}-\psi, \zeta \zeta\right)}{4 \pi \zeta^{2}(1-\psi)^{3}} .
$$

A useful parameter related to the motion of test particles around the structures on the equatorial plane is circular speed $v_{c}$ (rotation curves). For circular, equatorial orbits the 4-velocity $\mathbf{u}$ of the particles with respect to the coordinates frame has components $\mathbf{u}=u^{t}(1, \omega, 0,0)$, where $\omega=u^{\varphi} / u^{t}=\frac{d \varphi}{d t}$ is the angular speed of the test particles. With respect to tetrad (6a) - 6b , the 4-velocity has component

$$
u^{(a)}=e^{(a)}{ }_{b} u^{b}
$$

and the 3-velocity

$$
v^{(i)}=\frac{u^{(i)}}{u^{(t)}}=\frac{e^{(i)}{ }_{a} u^{a}}{e^{(t)}{ }_{a} u^{a}} .
$$

For circular, equatorial orbits the only nonvanishing velocity component is $v^{(\varphi)}$, and is given by

$$
\left[v^{(\varphi)}\right]^{2}=v_{c}^{2}=-\frac{g_{\varphi \varphi}}{g_{t t}} \omega^{2}
$$

and represents the circular speed (rotation profile) of the particle as seen by an observer at infinity.

The angular speed $\omega$ can be calculated considering the electrogeodesic motion of the particles. For the spacetime (11), the radial motion's equation is given by

$$
\frac{1}{2} g_{a b, R} u^{a} u^{b}=-\tilde{e} F_{R a} u^{a}
$$

where $\tilde{e}$ is the specific electric charge of the particles. For the electrostatic case we have

$$
\frac{1}{2} u^{0}\left(g_{\varphi \varphi, R} \omega^{2}+g_{t t, R}\right)=-\tilde{e} \phi, R
$$

where $u^{0}$ obtains normalizing $u^{a}$, that is requiring $g_{a b} u^{a} u^{b}=-1$, so that

$$
\left(u^{0}\right)^{2}=-\frac{1}{g_{\varphi \varphi} \omega^{2}+g_{t t}} .
$$


Thus, the angular speed $\omega$ is given by

$$
\omega^{2}=\frac{-T_{1} \pm \sqrt{T_{1}^{2}-4 T_{2} T_{3}}}{2 T_{2}},
$$

where

$$
\begin{aligned}
& T_{1}=2 g_{t t, R} g_{\varphi \varphi, R}+4 \tilde{e}^{2} g_{\varphi \varphi} \phi_{, R}^{2}, \\
& T_{2}=g_{\varphi \varphi, R}^{2} \\
& T_{3}=g_{t t, R}^{2}+4 \tilde{e}^{2} g_{t t} \phi_{, R}^{2} .
\end{aligned}
$$

The positive sign corresponds to the direct orbits or co-rotating and the negative sign to the retrograde orbits or counter-rotating.

To analyze the stability of the particles against radial perturbations we can use an extension of the Rayleigh criteria of stability of a fluid in rest in a gravitational field

$$
\frac{d\left(h^{2}\right)}{d R}>0
$$

where $h$ is the specific angular momentum, defined as $h=g_{\varphi a} u^{a}$. For circular, planar orbits we obtain

$$
h^{2}=\frac{R^{2}(1-\psi)^{2} v_{c}^{2}}{1-v_{c}^{2}} .
$$

All above quantities are evaluated on the equatorial plane $z=0$.

\section{CHARGED DUST THICK DISKS FROM A EXTREME REISSNER-NORDSTRÖM BLACK HOLE}

For a extreme Reissner-Nordström black hole

$$
\psi_{B H}=-\frac{M}{\sqrt{R^{2}+z^{2}}}
$$

and the application of the Miyamoto-Nagai transformation yield the potential

$$
\psi_{D}=-\frac{M}{\sqrt{R^{2}+\left(a+\sqrt{z^{2}+b^{2}}\right)^{2}}},
$$

which corresponds in Newtonian gravity to the first Miyamoto-Nagai model (with $G=1$ ). The relativistic energy density is given by

$$
\tilde{\rho}=\frac{\tilde{b}^{2}\left[\tilde{a} \tilde{R}^{2}+\left(\tilde{a}+3 \sqrt{\tilde{z}^{2}+\tilde{b}^{2}}\right)\left(\tilde{a}+\sqrt{\tilde{z}^{2}+\tilde{b}^{2}}\right)^{2}\right]}{4 \pi\left[\tilde{R}^{2}+\left(\tilde{a}+\sqrt{\tilde{z}^{2}+\tilde{b}^{2}}\right)^{2}\right]\left[1+\sqrt{\tilde{R}^{2}+\left(\tilde{a}+\sqrt{\tilde{z}^{2}+\tilde{b}^{2}}\right)^{2}}\right]^{3}\left(\tilde{z}^{2}+\tilde{b}^{2}\right)^{3 / 2}},
$$

where $\tilde{\rho}=M^{2} \rho, \tilde{R}=R / M, \tilde{z}=z / M, \tilde{a}=a / M$ and $\tilde{b}=b / M$. Note that the energy density is always a positive quantity in agreement with the weak energy condition.

In Figure 1 we graph the relativistic energy density $\tilde{\rho}$ and the contour curves for extreme Reissner-Nordström-like charged dust thick disks with parameters $\tilde{a}=1(a=M), \tilde{b}=1$ and 2 , as functions of $\tilde{R}$ and $\tilde{z}$. The energy density presents a maximum value on $\tilde{R}=0$ and then decreases rapidly with $\tilde{R}$ which permits to define a cut off radius $\tilde{R}_{c}$ and, in principle, to model these matter distributions as compact objects. Furthermore, as in the Newtonian case, as the ratio $b / a$ decreases the distribution of energy becomes flatter so that $b / a$ is also a measure of flatness of the models.

In figure 2 we illustrate the behavior of the circular speed for direct orbits $v_{+}^{2}$ and retrograde orbits $v_{-}^{2}$ of charged test particles with $\tilde{e}=0$ (neutral particles), $0.5,0.9$ and the same values of the parameters $\tilde{a}$ and $\tilde{b}$. We find that for direct orbits the specific electric charge increases the speed of the particles whereas for retrograde orbits the contrary occurs. Also, for direct orbit the particles become more relativistic when the matter distribution is flatted, whereas for retrograde orbits the opposite occurs. We observer that circular speed of particles is always a quantity less than the speed of light in agreement with the dominant energy condition. In figure 3 we also show, as function of $\tilde{R}$, the specific angular momentums $\tilde{h}_{+}^{2}$ and $\tilde{h}_{-}^{2}$ for the same values of parameters. We find that for these values the orbits of the particles are stable against radial perturbation. 


\section{MORGAN-MORGAN-LIKE CHARGED DUST THICK DISKS}

Thin disks of finite extension can be obtained in Newtonian gravity solving the Laplace equation in oblate spheroidal coordinates $(u, v)$, which are defined in terms of the cylindrical coordinates $(R, z)$ by

$$
\begin{aligned}
R^{2} & =d^{2}\left(1+u^{2}\right)\left(1-v^{2}\right), \\
z & =d u v
\end{aligned}
$$

and explicitly

$$
\begin{aligned}
& \sqrt{2} u=\sqrt{\left[\left(\tilde{R}^{2}+\tilde{z}^{2}-1\right)^{2}+4 \tilde{z}^{2}\right]^{1 / 2}+\tilde{R}^{2}+\tilde{z}^{2}-1}, \\
& \sqrt{2} v=\sqrt{\left[\left(\tilde{R}^{2}+\tilde{z}^{2}-1\right)^{2}+4 \tilde{z}^{2}\right]^{1 / 2}-\left(\tilde{R}^{2}+\tilde{z}^{2}-1\right)},
\end{aligned}
$$

where $u \geq 0,-1<v<1, \tilde{R}=R / d$ and $\tilde{z}=z / d$, being $d$ the radius of the disk.

In such coordinates, the general solution of Laplaces equation can be written as

$$
\Phi=-\sum_{n=0}^{\infty} c_{2 n} q_{2 n}(u) P_{2 n}(v),
$$

where $c_{2 n}$ are constants, $P_{2 n}$ are the Legendre polynomials of order $2 n$ and

$$
q_{2 n}(u)=i^{2 n+1} Q_{2 n}(i u)
$$

being $Q_{2 n}(i u)$ the Legendre functions of the second kind. For example, for the first two terms in series (23) $(n=0$ and $n=1$ ), the gravitational potential is

$$
\Phi=-\frac{M G}{d}\left\{\cot ^{-1}(u)+\frac{1}{4}\left[\left(3 u^{2}+1\right) \cot ^{-1}(u)-3 u\right]\left(3 v^{2}-1\right)\right\}
$$

being $M$ the mass of the disk.

Note that in general the potential $\Phi$ is function of $z^{2}=|z|^{2}$. Thus, Newtonian thick disks can be generated from the solutions (23) via a Miyamoto-Nagai transformation taking $a=0$. In particular, for the potential (25) we obtain the mass distribution

$$
\tilde{\rho}_{N}=\frac{3 \tilde{b}^{2}}{4 \pi \tilde{d}^{3}\left(x^{2}+y^{2}\right) x^{3}}
$$

where $\tilde{\rho}=M^{2} \rho, \tilde{d}=d / M, \tilde{b}=b / d$, and

$$
\begin{aligned}
& \sqrt{2} x=\sqrt{\left[\left(\tilde{R}^{2}+\tilde{\zeta}^{2}-1\right)^{2}+4 \tilde{\zeta}^{2}\right]^{1 / 2}+\tilde{R}^{2}+\tilde{\zeta}^{2}-1}, \\
& \sqrt{2} y=\sqrt{\left[\left(\tilde{R}^{2}+\tilde{\zeta}^{2}-1\right)^{2}+4 \tilde{\zeta}^{2}\right]^{1 / 2}-\left(\tilde{R}^{2}+\tilde{\zeta}^{2}-1\right)},
\end{aligned}
$$

where $\tilde{\zeta}=\sqrt{\tilde{z}^{2}+\tilde{b}^{2}}$.

The relativistic version of the disk like solutions (23) was first given in [2] and are known in the literature as the Morgan-Morgan disks. Likewise, relativistic finite thin disks make of charged dust can be constructed taking the Newtonian gravitational potential $\Phi$ as the metric function $\psi$ and in consequence can also be "fattened" by applying a Miyamoto-Nagai transformation. Thus, for the seed potential (25) we obtain the relativistic energy density (with $\mathrm{G}=1)$

$$
\tilde{\rho}=\frac{3 \tilde{b}^{2}}{4 \pi \tilde{d}^{3}\left(x^{2}+y^{2}\right) x^{3}(1-\psi)^{3}}
$$

where

$$
\psi=-\frac{1}{\tilde{d}}\left\{\cot ^{-1}(x)+\frac{1}{4}\left[\left(3 x^{2}+1\right) \cot ^{-1}(x)-3 x\right]\left(3 y^{2}-1\right)\right\}
$$


In figure 4 we present, as functions of $\tilde{R}$ and $\tilde{z}$, the surface and level curves of the energy density $\tilde{\rho}$ for MorganMorgan-like charged dust thick disks with parameters $\tilde{d}=1, \tilde{b}=1$ and $\tilde{b}=2$. We see that the energy density is a positive quantity in according with the weak energy condition and, unlike the seed thin disks, these disks have no boundary but as the distribution of matter also decreases rapidly with radius $\tilde{R}$ it permits to model these structures as compact objects. Since the denominator of the expression of the energy density is a quantity greater than one, the relativistic effects decrease everywhere the energy density. Here the parameter $\tilde{b} / \tilde{d}$ is the measure of flatness of the models.

In figure [5 we graph the circular speed for direct orbits $v_{+}^{2}$ and retrograde orbits $v_{-}^{2}$ for charged test particles moving around Morgan-Morgan-like charged dust thick disks with parameters $\tilde{e}=0$ (neutral particles), $0.5,0.9$ and the same values of the parameter $\tilde{d}$ and $\tilde{b}$, as functions of $\tilde{R}$. We see that they exhibit the same behavior as the previous models. However, we observer that in these models the particles are more relativistic in the case of direct orbits, whereas for retrograde orbit the opposite occurs. Note also that circular speed of particles is always a quantity less than the speed of light in agreement with the dominant energy condition. The orbits of particles are also stable against radial perturbations for these values of parameters (figure 6).

\section{MORGAN-MORGAN-LIKE CHARGED DUST THICK RINGS}

Ring like matter distributions can be obtained using a Kelvin transformation [37, 38] which in cylindrical coordinates reads

$$
(R, z) \rightarrow\left(\frac{d^{2} R}{R^{2}+z^{2}}, \frac{d^{2} z}{R^{2}+z^{2}}\right) .
$$

When this transformation is applied to the relativistic potential-density pair $(\psi, \rho)$ corresponding to the non-linear Poisson's equation (5b) we obtain the new pair

$$
\begin{aligned}
& \bar{\psi}=\left(\frac{d}{\sqrt{R^{2}+z^{2}}}\right) \psi\left(\frac{d^{2} R}{R^{2}+z^{2}}, \frac{d^{2} z}{R^{2}+z^{2}}\right), \\
& \bar{\rho}=\left(\frac{d^{5}}{\left(R^{2}+z^{2}\right)^{5 / 2}}\right) \rho\left(\frac{d^{2} R}{R^{2}+z^{2}}, \frac{d^{2} z}{R^{2}+z^{2}}\right) .
\end{aligned}
$$

In particular, for the potential-density pair (28) - (29) we obtain

$$
\begin{aligned}
\tilde{\rho} & =\frac{3 \tilde{b}^{2}}{4 \pi \tilde{d}^{3} \tilde{r}^{5}\left(\bar{x}^{2}+\bar{y}^{2}\right) \bar{x}^{3}(1-\bar{\psi})^{3}}, \\
\bar{\psi} & =-\frac{1}{\tilde{d} \tilde{r}}\left\{\cot ^{-1}(\bar{x})+\frac{1}{4}\left[\left(3 \bar{x}^{2}+1\right) \cot ^{-1}(\bar{x})-3 \bar{x}\right]\left(3 \bar{y}^{2}-1\right)\right\},
\end{aligned}
$$

where again $\tilde{\rho}=M^{2} \bar{\rho}, \tilde{d}=d / M, \tilde{b}=b / d$,

$$
\begin{aligned}
& \sqrt{2} \tilde{r} \bar{x}=\sqrt{\left[\left(1+\left(\tilde{b}^{2}-1\right) \tilde{r}^{2}\right)^{2}+4\left(\tilde{z}^{2}+\tilde{b}^{2} \tilde{r}^{4}\right)\right]^{1 / 2}+1+\left(\tilde{b}^{2}-1\right) \tilde{r}^{2}} \\
& \sqrt{2} \tilde{r} \bar{y}=\sqrt{\left[\left(1+\left(\tilde{b}^{2}-1\right) \tilde{r}^{2}\right)^{2}+4\left(\tilde{z}^{2}+\tilde{b}^{2} \tilde{r}^{4}\right)\right]^{1 / 2}-1+\left(1-\tilde{b}^{2}\right) \tilde{r}^{2}} .
\end{aligned}
$$

and $\tilde{r}=\sqrt{\tilde{R}^{2}+\tilde{z}^{2}}$, being $\tilde{R}=R / d$ and $\tilde{z}=z / d$.

In figure 7 we show, as functions of $\tilde{R}$ and $\tilde{z}$, the density profile $\tilde{\rho}$ and contour plots for Morgan-Morgan-like charged dust thick rings with parameters $\tilde{d}=1, \tilde{b}=0.2$ and $\tilde{b}=0.5$. The graphs suggest that we have a ring structure. In all cases we find that the energy density is a positive quantity. In figure 8 we present the tangential speeds $v_{+}^{2}$ and $v_{-}^{2}$ for charged test particles orbiting on the equatorial plane and their respective specific angular momentums $h_{+}^{2}$ and $h_{-}^{2}$ for parameters $\tilde{d}=1$ and $\tilde{b}=0.5$. These quantities present the same behavior that in the previous models. However, for lower values of $\tilde{b}$, for example $\tilde{b}=0.2$, one finds a central region where the particles present superluminal or tachyonic speeds and the orbits are unstable.

\section{DISCUSSION}

Two simple families of charged dust thick disks and a family of thick annular disks for a particular conformastatic metric were presented based on the extreme Reissner-Nordstrom black hole field and a Morgan-Morgan-like metric. 
The disk models are constructed via a Miyamoto-Nagai transformation used in Newtonian gravity to model flat galaxies and the ring like matter distributions applying a Kelvin transformation. The structures satisfy all the energy conditions. Unlike the Morgan-Morgan-like thin disks, the relativistic thick disks built from them have infinite extension, but as the energy density decreases rapidly with radius $\tilde{R}$ we can to model such structures as compact objects.

We analyzed the electrogeodesic equatorial circular motion of charged test particles around of the disks. We found that for direct orbits the specific electric charge increases the speed of the particles whereas for retrograde orbits the contrary occurs. Also, for direct orbit the particles become more relativistic when the matter distribution is flatted, whereas for retrograde orbits the opposite occurs. In the Morgan-Morgan-like disk models the speed of the particles is greater in the case of direct orbits, whereas for retrograde orbit the opposite occurs. In all the cases, we found stable orbits against radial perturbations.

\section{REFERENCES}

[1] W. A. Bonnor and A. Sackfield, Commun. Math. Phys. 8, 338 (1968).

[2] T. Morgan and L. Morgan, Phys. Rev. 183, 1097 (1969).

[3] L. Morgan and T. Morgan, Phys. Rev. D 2, 2756 (1970).

[4] D. Lynden-Bell and S. Pineault, Mon. Not. R. Astron. Soc. 185, 679 (1978).

[5] A. Chamorro, R. Gregory, and J. M. Stewart, Proc. R. Soc. London A413, 251 (1987).

[6] P.S. Letelier and S. R. Oliveira, J. Math. Phys. 28, 165 (1987).

[7] J. P. S. Lemos, Class. Quantum Grav. 6, 1219 (1989).

[8] J. Bičák, D. Lynden-Bell, and J. Katz, Phys. Rev. D 47, 4334 (1993).

[9] J. Bičák, D. Lynden-Bell, and C. Pichon, Mon. Not. R. Astron. Soc. 265, 126 (1993).

[10] G. A. González and O. A. Espitia, Phys. Rev. D 68, 104028 (2003).

[11] J. Bičák and T. Ledvinka, Phys. Rev. Lett. 71, 1669 (1993).

[12] G. A. González and P. S. Letelier, Phys. Rev. D 62, 064025 (2000).

[13] J. P. S. Lemos and P. S. Letelier, Class. Quantum Grav. 10, L75 (1993).

[14] J. P. S. Lemos and P. S. Letelier, Phys. Rev. D 49, 5135 (1994).

[15] G. A. González and P. S. Letelier, Phys. Rev. D 69, 044013 (2004).

[16] D. Vogt and P. S. Letelier, Phys. Rev. D 76, 084010 (2007).

[17] T. Ledvinka, J. Bičák, and M. Žofka, in Proceeding of 8th Marcel-Grossmann Meeting in General Relativity, edited by T. Piran (World Scientific, Singapore, 1999)

[18] J. Katz, J. Bičák, and D. Lynden-Bell, Class. Quantum Grav. 16, 4023 (1999).

[19] P. S. Letelier, Phys. Rev. D 60, 104042 (1999).

[20] G. García R. and G. A. González, Phys. Rev. D 69, 124002 (2004).

[21] G. García-Reyes and G. A. González, Class. Quantum Grav. 21, 4845 (2004).

[22] C. H. García-Duque and G. García-Reyes, Gen. Relativ. Gravit. 43, 11, 3001 (2011).

[23] G. García-Reyes and O. A. Espitia, Gen. Relativ. Gravit. 46, 1674 (2014).

[24] S. Rosseland, Mon. Not. R. Astron. Soc. 84, 720 (1924).

[25] J. Bally and E. R. Harrison, Astrophys. J. 220, 743 (1978).

[26] D. Vogt and P. S. Letelier, Phys. Rev. D 70, 064003 (2004).

[27] S. D. Majumdar, Phys. Rev. 72, 390 (1947).

[28] A. Papapetrou, Proc. Roy. Soc. (London) A51, 191 (1947).

[29] W. A. Bonnor, Class. Quantum Grav. 15, 351 (1998).

[30] D. Vogt and P. S. Letelier, Class. Quantum Grav. 21, 3369 (2004).

[31] G. A. González, A. C. Gutiérrez-piñeres and P. A. Ospina, Phys. Rev. D 78, 064058 (2008).

[32] F. D. Lora-Clavijo, P. A. Ospina-Henao and J. F. Pedraza, Phys. Rev. D 82, 084005 (2010).

[33] M. Miyamoto and R. Nagai, PASJ 27, 533 (1975).

[34] R. Nagai and M. Miyamoto, PASJ 28, 1 (1976).

[35] G. G. Kuzmin 1956, Astron. Zh. 33, 27 (1956).

[36] A. Toomre, Ap. J. 138, 385 (1962).

[37] W. Thomson (Lord Kelvin), J. Math. Pures Appliquees 12, 256 (1847).

[38] O. D. Kellog, Foundations of Potential Theory, Dover Publications, New York (1953). 


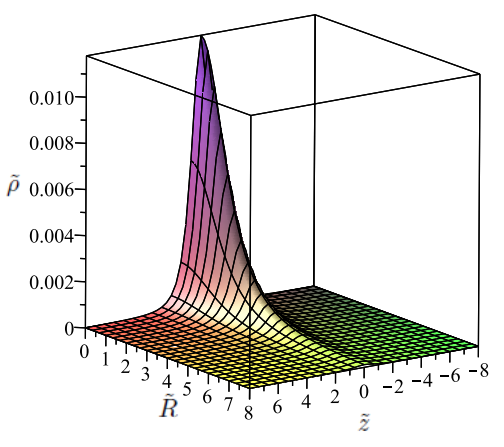

(a)

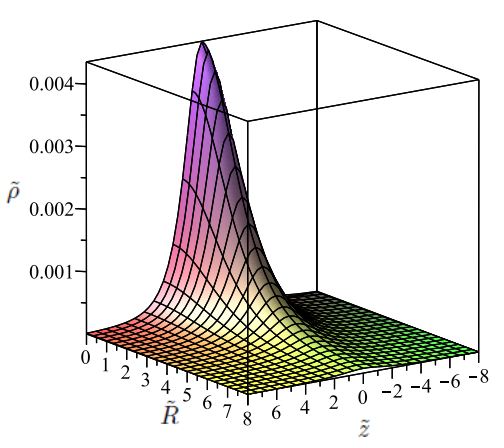

$(c)$

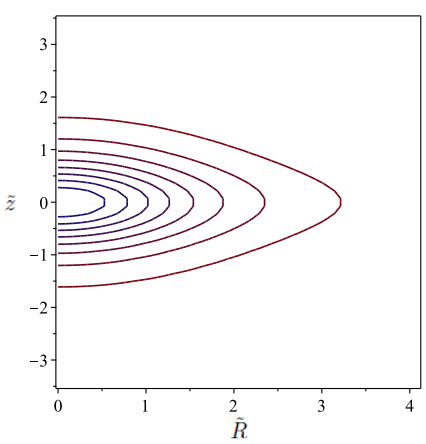

(b)

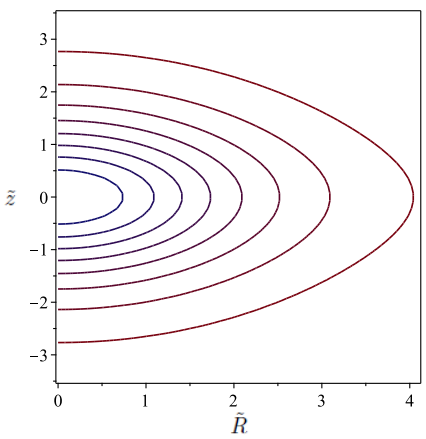

$(d)$

FIG. 1. The relativistic energy density $\tilde{\rho}$ and contour plots for extreme Reissner-Nordström-like charged dust thick disks with parameters $\tilde{a}=1, \tilde{b}=1$ (top figures) and $\tilde{b}=2$, as functions of $\tilde{R}$ and $\tilde{z}$. 

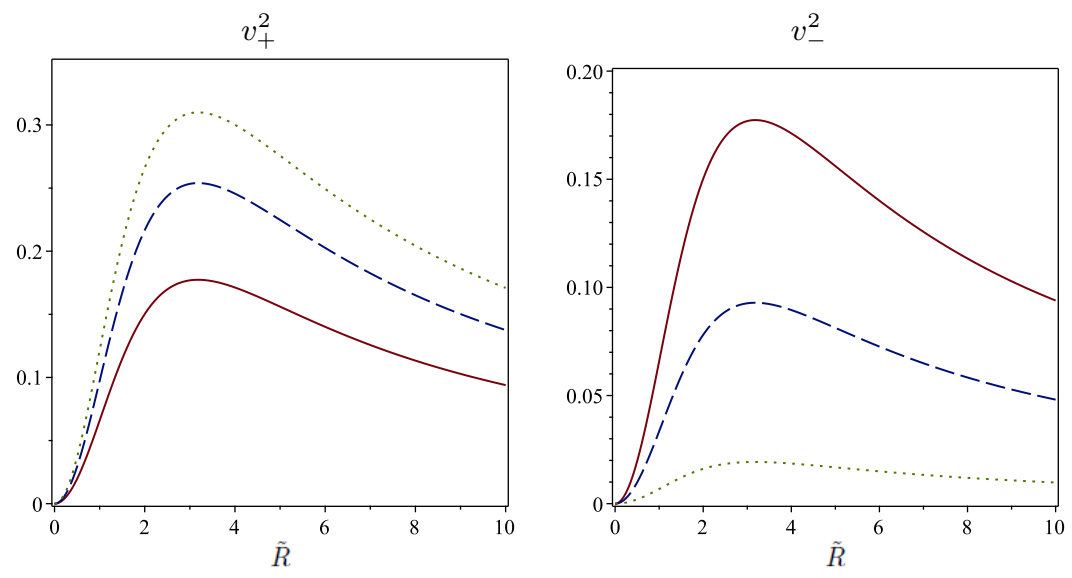

(a)

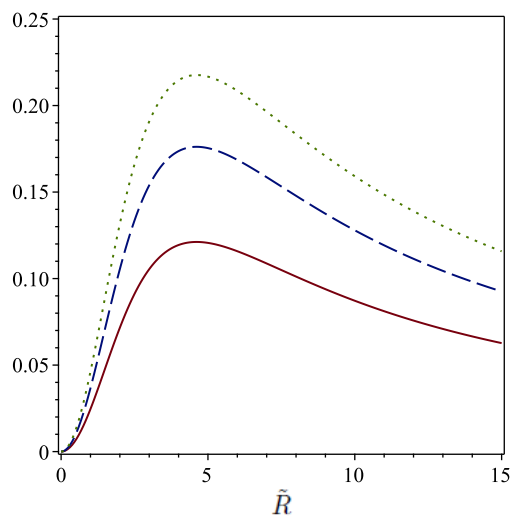

(b)

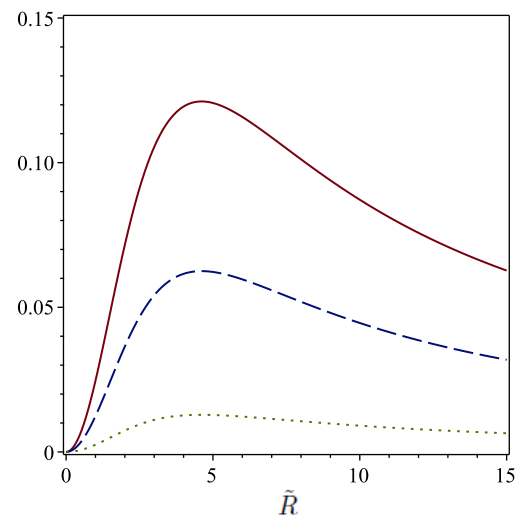

$(d)$

FIG. 2. The circular speed $v_{+}^{2}$ and $v_{-}^{2}$ for charged test particles around extreme Reissner-Nordström-like charged dust thick disks with parameters $\tilde{e}=0$ (solid curves), $0.5,0.9$ (dotted curves), $\tilde{a}=1, \tilde{b}=1$ (top figures) and $\tilde{b}=2$, as functions of $\tilde{R}$ 

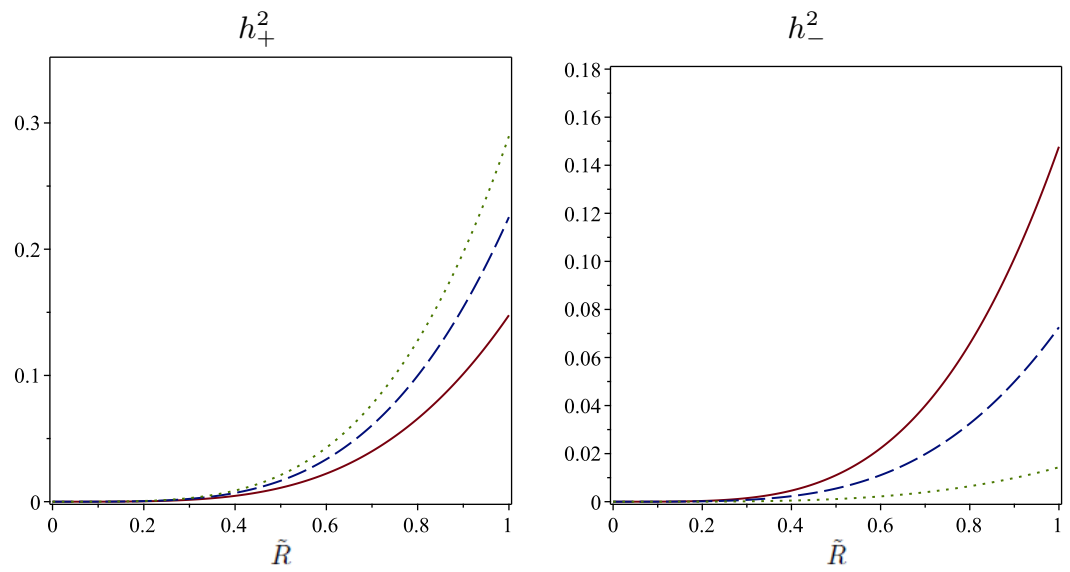

(a)

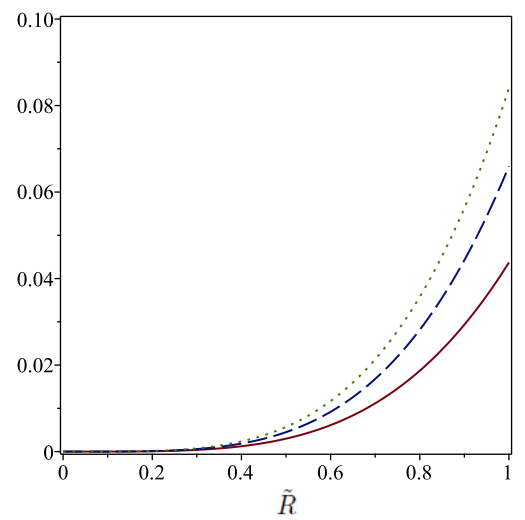

(b)



(c)

(d)

FIG. 3. The specific angular momentum $h_{+}^{2}$ and $h_{-}^{2}$ for charged test particles around extreme Reissner-Nordström-like charged dust thick disks with parameters $\tilde{e}=0$ (solid curves), 0.5, 0.9 (dotted curves), $\tilde{a}=1, \tilde{b}=1$ (top figures) and $\tilde{b}=2$, as functions of $\tilde{R}$ 


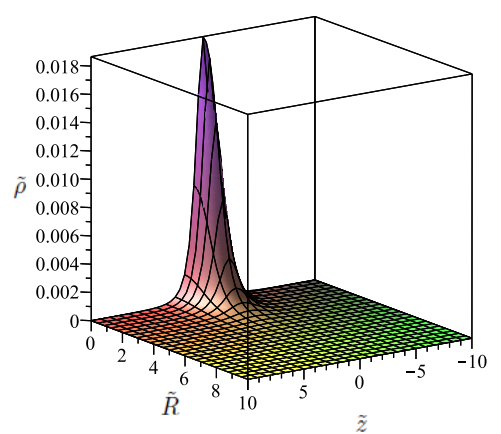

(a)

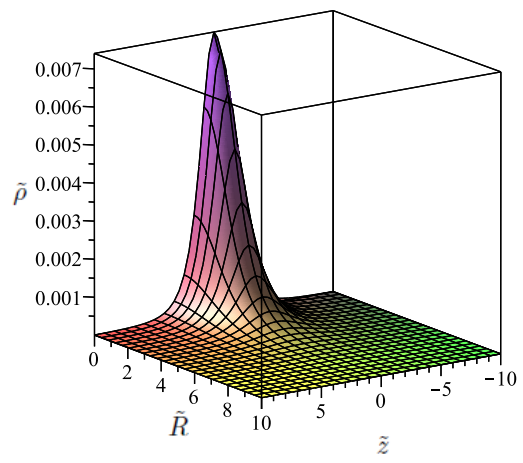

(c)

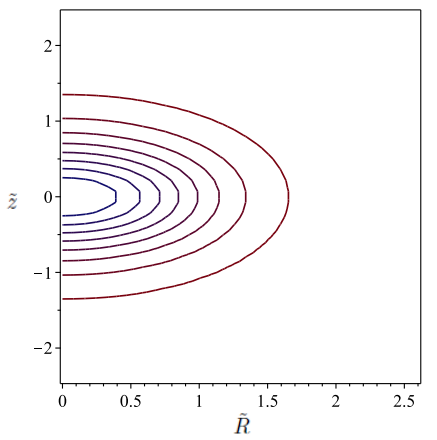

(b)

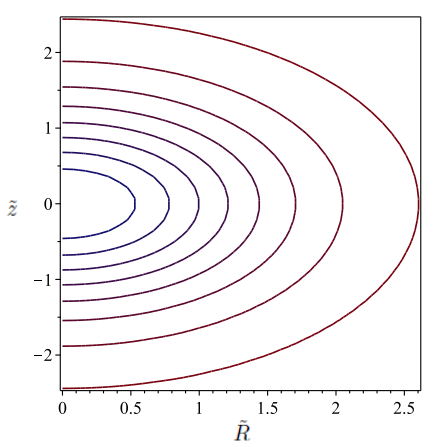

(d)

FIG. 4. The relativistic energy density $\tilde{\rho}$ and contour curves for Morgan-Morgan-like charged dust thick disks with parameters $\tilde{d}=1, \tilde{b}=1$ (top figures) and $\tilde{b}=2$, as functions of $\tilde{R}$ and $\tilde{z}$. 



(a)

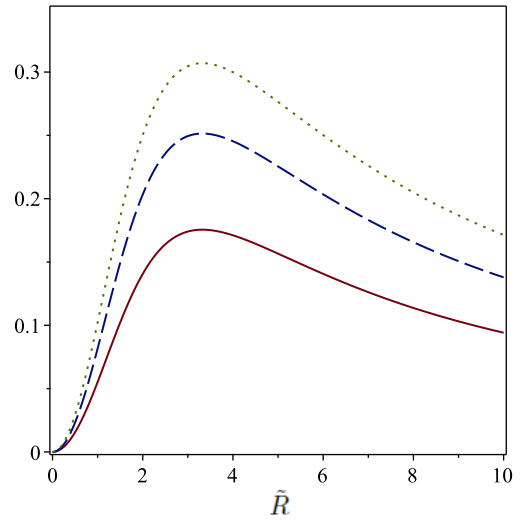

(c) (b)

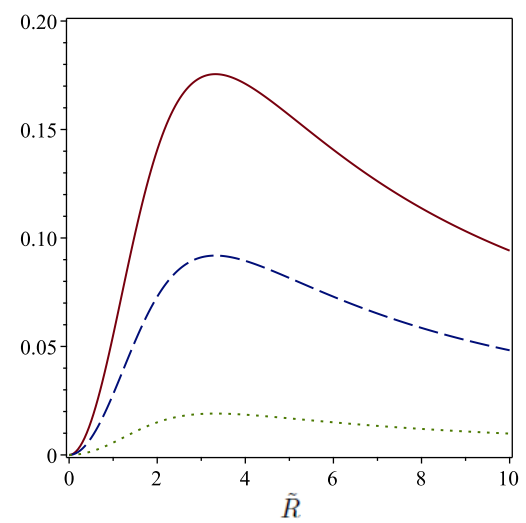

(d)

FIG. 5. The circular speed $v_{+}^{2}$ and $v_{-}^{2}$ for charged test particles around Morgan-Morgan-like charged dust thick disks with parameters $\tilde{e}=0$ (solid curves), $0.5,0.9$ (dotted curves), $\tilde{d}=1, \tilde{b}=1$ (top figures) and $\tilde{b}=2$, as functions of $\tilde{R}$ 

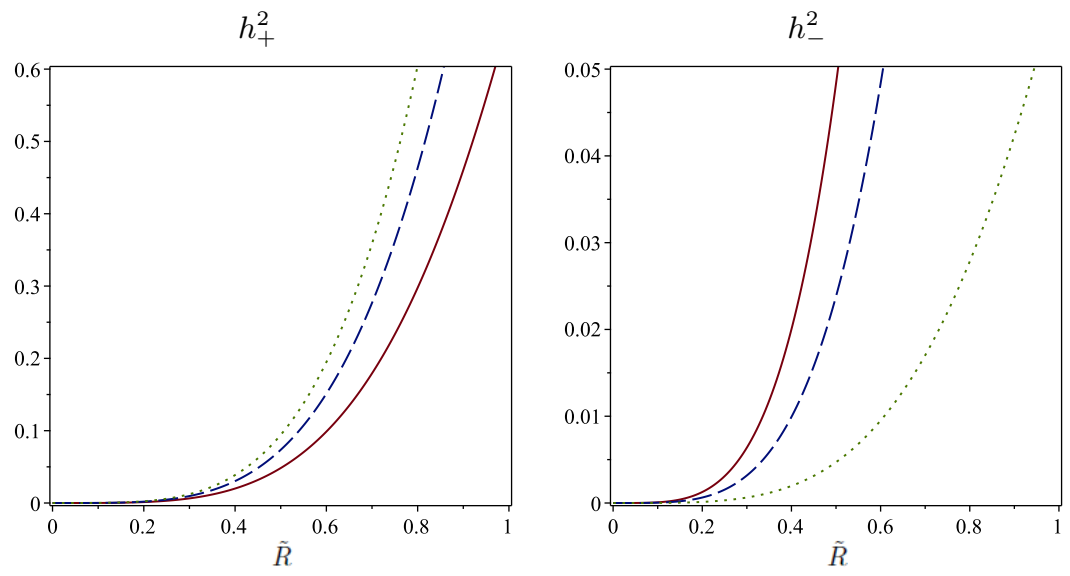

(a)

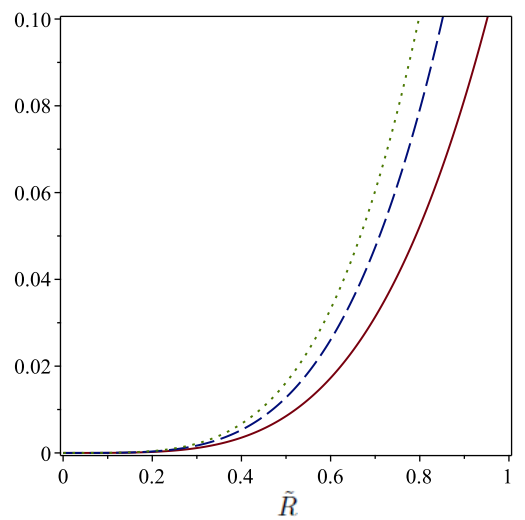

$(b)$

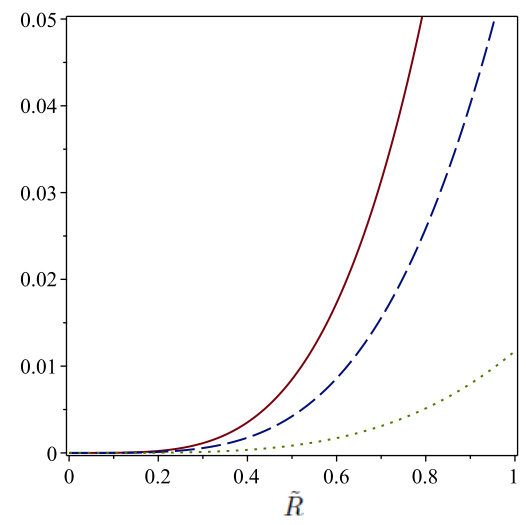

$(c)$

$(d)$

FIG. 6. The specific angular momentum $h_{+}^{2}$ and $h_{-}^{2}$ for charged test particles around Morgan-Morgan-like charged dust thick disks with parameters $\tilde{e}=0$ (solid curves), $0.5,0.9$ (dotted curves), $\tilde{d}=1, \tilde{b}=1$ (top figures) and $\tilde{b}=2$, as functions of $\tilde{R}$ 


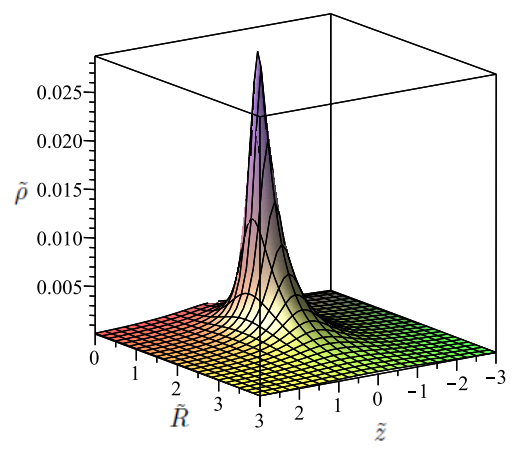

(a)

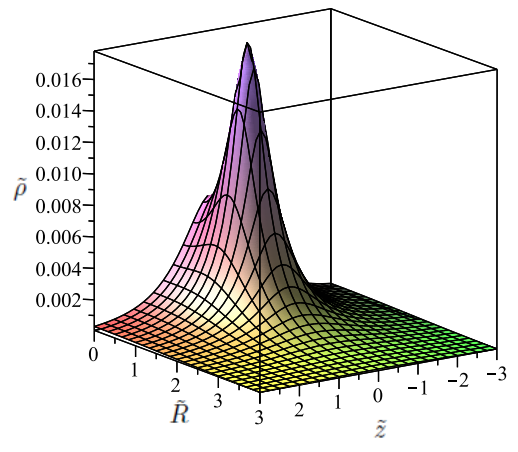

$(c)$

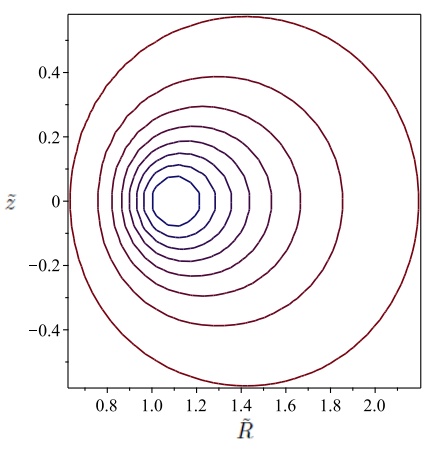

(b)

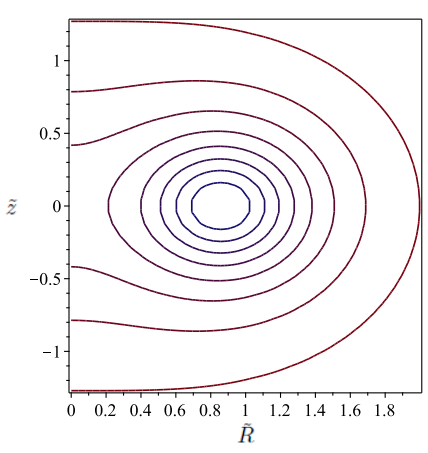

$(d)$

FIG. 7. The surface and level curves of the relativistic energy density $\tilde{\rho}$ for Morgan-Morgan-like charged dust thick rings with parameters $\tilde{d}=1, \tilde{b}=0.2$ (top figures) and $\tilde{b}=0.5$, as functions of $\tilde{R}$ and $\tilde{z}$. 


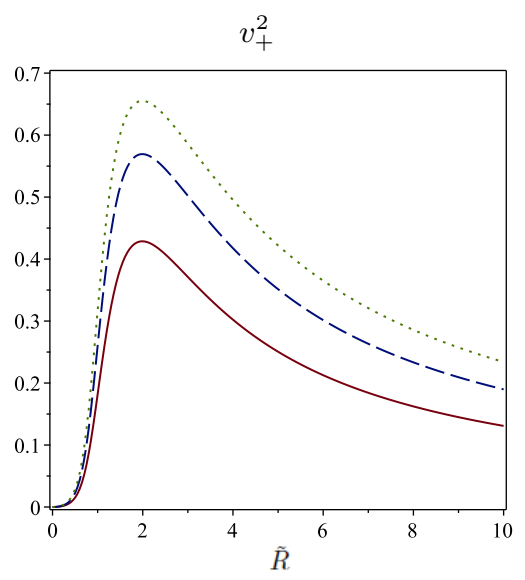

(a)

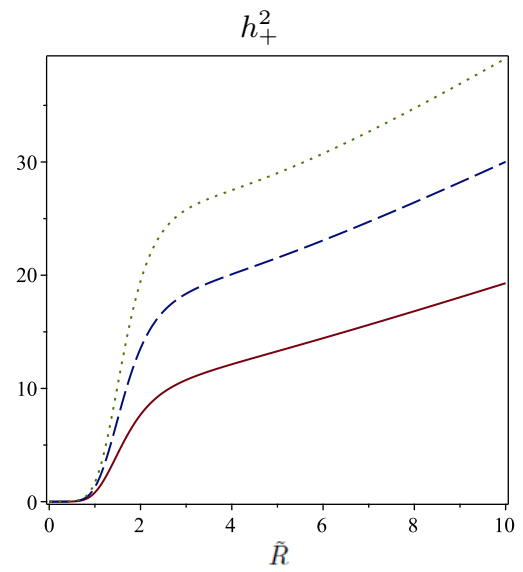

(c)

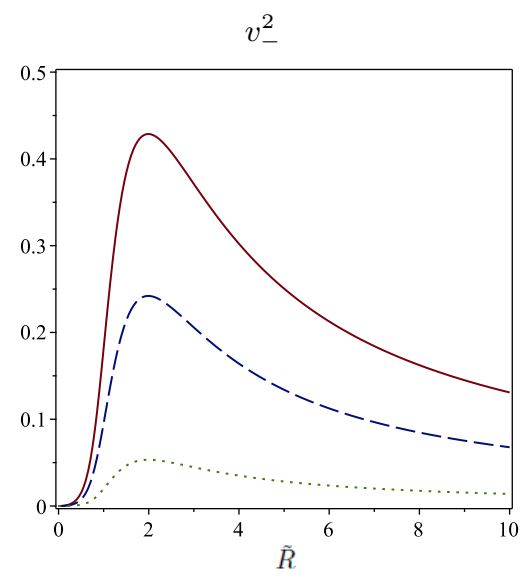

(b)

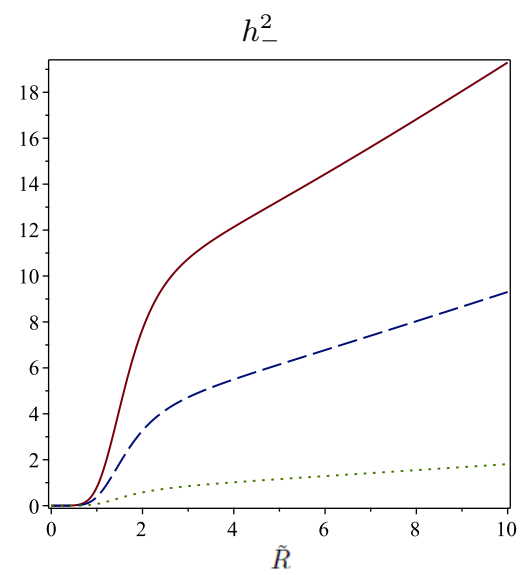

(d)

FIG. 8. The circular speed $v_{+}^{2}$ and $v_{-}^{2}$ (top curves) and the specific angular momentum $h_{+}^{2}$ and $h_{-}^{2}$ for charged test particles around Morgan-Morgan-like charged dust thick rings with parameters $\tilde{e}=0$ (solid curves), $0.5,0.9$ (dotted curves), $\tilde{d}=1$, $\tilde{b}=0.5$, as functions of $\tilde{R}$ 